\title{
Magnetic Trapping and Coherent Control of Laser-Cooled Molecules
}

\author{
H. J. Williams, L. Caldwell, N. J. Fitch, S. Truppe, J. Rodewald, E. A. Hinds, B. E. Sauer, and M. R. Tarbutt \\ Centre for Cold Matter, Blackett Laboratory, Imperial College London, Prince Consort Road, London SW7 2AZ, United Kingdom
}

(Received 5 December 2017; published 16 April 2018)

\begin{abstract}
We demonstrate coherent microwave control of the rotational, hyperfine, and Zeeman states of ultracold $\mathrm{CaF}$ molecules, and the magnetic trapping of these molecules in a single, selectable quantum state. We trap about $5 \times 10^{3}$ molecules for almost $2 \mathrm{~s}$ at a temperature of $70(8) \mu \mathrm{K}$ and a density of $1.2 \times 10^{5} \mathrm{~cm}^{-3}$. We measure the state-specific loss rate due to collisions with background helium.
\end{abstract}

DOI: 10.1103/PhysRevLett.120.163201

Techniques for producing and controlling ultracold molecules are advancing rapidly, motivated by a wide range of applications. These include precise measurements that test fundamental physics [1,2], quantum state resolved collisions and chemistry [3], quantum computation [4-6] and simulation $[7,8]$, and the study of dipolar quantum gases [9]. These applications call for trapped molecules in a single, selectable quantum state, and they typically require coherent control over the rotational and hyperfine states. Such control has recently been achieved [10-13] for bialkali molecules produced by associating ultracold atoms [14-18]. Great efforts are also being made to cool molecules directly, for example, by optoelectrical Sisyphus cooling [19] or direct laser cooling and magneto-optical trapping [20-25]. These methods can produce ultracold molecules with greater chemical diversity and with large electric and magnetic dipoles, as is often desired. The magneto-optical trap (MOT) is an excellent tool for collecting and cooling molecules, and it promises to be the starting point for many applications of ultracold molecules, just as it has been for ultracold atoms. However, it does not allow quantum state control, provides limited phase-space density, and has a limited lifetime due to optical pumping into states not addressed by the lasers. Thus, molecules in a MOT must be transferred into a conservative trap where the lifetime can be long, the quantum state can be selected and preserved, and the phase-space density can be increased, for example, by sympathetic, evaporative, or Raman sideband cooling. Magnetic traps have been crucial for exploiting ultracold atoms, and they have previously been used to confine molecules produced at $\sim 100 \mathrm{mK}$ by buffer-gas cooling and Stark and Zeeman deceleration [26-33]. Here, we

Published by the American Physical Society under the terms of the Creative Commons Attribution 4.0 International license. Further distribution of this work must maintain attribution to the author(s) and the published article's title, journal citation, and DOI. demonstrate coherent control and magnetic trapping of laser-cooled molecules, which are key steps towards the applications discussed above. Starting from a MOT of $\mathrm{CaF}$ [25], we compress the cloud to increase its density, cool the molecules to sub-Doppler temperature [23], optically pump them into a single internal state, transfer them coherently to a selectable rotational, hyperfine and Zeeman level, and then confine them in a magnetic trap.

Our setup is the same as used previously [23,25], with the addition of microwave components to drive the rotational transition. A pulse of $\mathrm{CaF}$ emitted at time $t=0$ from a cryogenic buffer gas source [34] is decelerated by frequency-chirped counter-propagating laser light [35]. The slowest molecules are captured in a dual-frequency MOT $[25,36]$, where the main laser drives the $A^{2} \Pi_{1 / 2}(v=0$, $J=1 / 2) \leftarrow X^{2} \Sigma^{+}(v=0, N=1)$ transition, with intensity $I$ and detuning $\delta$. The linewidth of this transition is $\Gamma=2 \pi \times 8.3 \mathrm{MHz}$. Three additional lasers repump the population that leaks to the $v=1,2$, and 3 vibrational levels of $X^{2} \Sigma^{+}$. The MOT and magnetic trap share the same in-vacuum coils (see Sec. 2 of Ref. [25]), which produce an axial field gradient $B^{\prime}$. Using $I=I_{\max }=400 \mathrm{~mW} \mathrm{~cm}^{-2}$, $\delta=-0.75 \Gamma$, and $B^{\prime}=30 \mathrm{Gcm}^{-1}$, we routinely capture $2 \times 10^{4}$ molecules with a peak density of $n=6 \times 10^{5} \mathrm{~cm}^{-3}$ and a temperature, $T$, near $7 \mathrm{mK}$.

Figure 1(a) shows the energy levels most relevant to the present work, labeled $\left|N, F, M_{F}\right\rangle$. The ground rotational state, $N=0$, is split into two hyperfine components, $F=0,1$, while the first excited rotational state, $N=1$, is split by spin-rotation and hyperfine interactions into four components with $F \in\{1,0,1,2\}$. To address these, the MOT laser is tuned near $F=0$, the sideband of a $48 \mathrm{MHz}$ acousto-optic modulator (AOM) addresses the upper $F=1$ level and the sidebands of an electro-optic modulator (EOM) address the $F=2$ and lower $F=1$ levels. Since our previous work, we have changed the EOM frequency from $74.5 \mathrm{MHz}$ to $70.5 \mathrm{MHz}$. This has increased the density by a factor of 4 , mainly by increasing the MOT spring constant. 


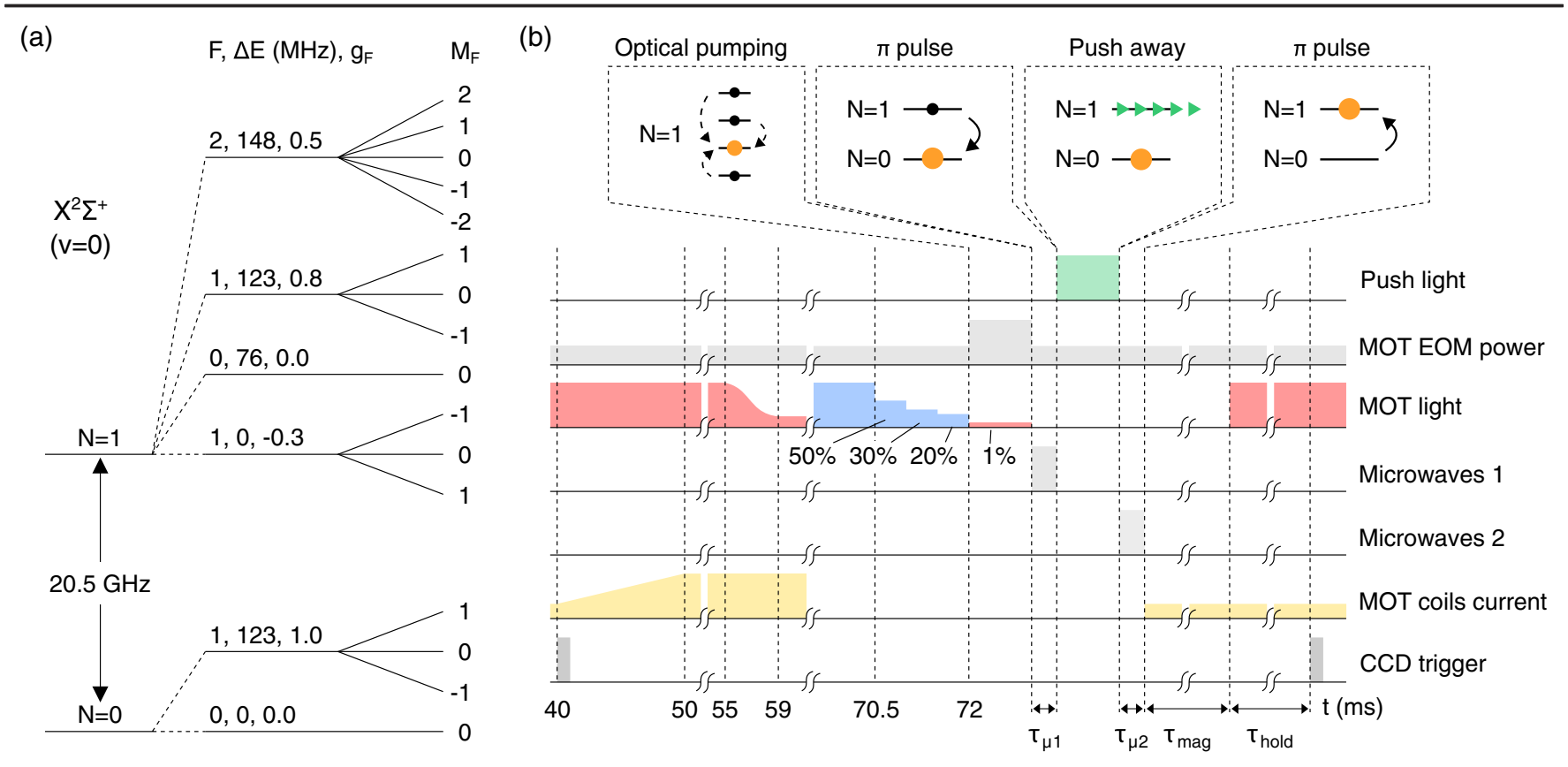

FIG. 1. (a) The energy levels (not to scale) of the two lowest-lying rotational states of $\mathrm{CaF}, X^{2} \Sigma^{+}(v=0)$. Hyperfine splittings and magnetic g-factors are given. (b) The timing sequence of the experiment (not to scale), starting from $t=40 \mathrm{~ms}$, when the first fluorescence image is taken. The MOT light sequence is color coded according to detuning. The MOT is first compressed by ramping up $B^{\prime}$. Then, the molecules are cooled in a blue-detuned molasses, optically pumped into $|1,0,0\rangle$, and transferred to $N=0$ with a microwave pulse. A push pulse removes those remaining in $N=1$, then (optionally) another microwave pulse transfers from $N=0$ back to $N=1$. Finally, the magnetic trap is turned on.

Figure 1(b) illustrates the new control steps we implement and presents their timings. Each sequence begins with a fluorescence image taken at $t=40 \mathrm{~ms}$, used to determine the number of molecules, $N_{\text {mol }}$, in each MOT. We first compress the MOT by increasing $B^{\prime}$ linearly between $t=$ 40 and $50 \mathrm{~ms}$ and holding the higher $B^{\prime}$ until $t=55 \mathrm{~ms}$. Figure 2 shows $n$ as a function of $B^{\prime}$ in the compressed MOT (CMOT). Increasing $B^{\prime}$ from 30 to $113 \mathrm{G} \mathrm{cm}^{-1}$ increases $n$ by a factor of 5.3. If $N_{\mathrm{mol}}$ and $T$ are conserved in the compression, we expect $n \propto\left(B^{\prime}\right)^{3 / 2}$ [37]. Fitting to the model $n=a\left(B^{\prime}\right)^{b}$ yields the less rapid scaling $b=1.16(1)$. We attribute this to $T$ increasing with $B^{\prime}$; we measure $T=7.36(9) \mathrm{mK}$ at $B^{\prime}=30 \mathrm{G} \mathrm{cm}^{-1}$, increasing to $8.75(5) \mathrm{mK}$ at $B^{\prime}=69 \mathrm{G} \mathrm{cm}^{-1}$. For all subsequent data, we use $B^{\prime}=69 \mathrm{G} \mathrm{cm}^{-1}$ in the CMOT, giving $n \approx 2 \times 10^{6} \mathrm{~cm}^{-3}$.

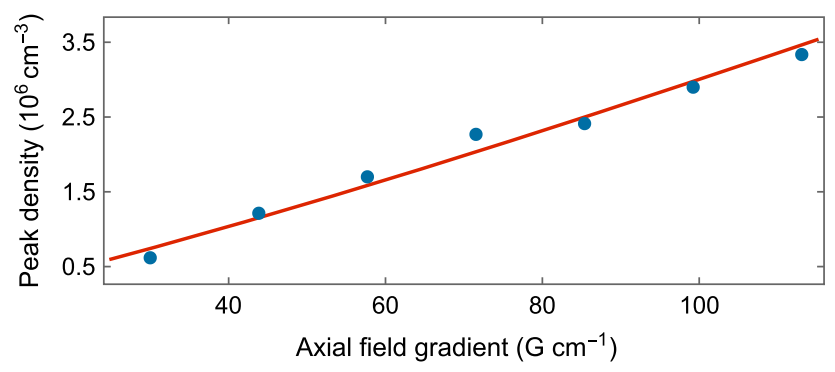

FIG. 2. The density versus axial magnetic field gradient used for the compressed MOT. Line: fit to $n=a\left(B^{\prime}\right)^{b}$.
Following the CMOT, we lower the temperature using a procedure similar to that described previously [23] and illustrated in Fig. 1(b). Between $t=55$ and $59 \mathrm{~ms}, I$ is ramped down to $0.1 I_{\max }$, where it is held until the MOT coils and laser are switched off at $t=63 \mathrm{~ms}$. At $t=64 \mathrm{~ms}$, the laser is re-enabled at full intensity but with $\delta=3 \Gamma$, realizing a blue-detuned optical molasses. After $6.5 \mathrm{~ms}, I$ is stepped downwards to 50,30 , and $20 \%$ of $I_{\max }$, with each value held for $0.5 \mathrm{~ms}$. This procedure lowers $T$ to $55 \mu \mathrm{K}$. The molasses duration is far longer than the $1 \mathrm{~ms}$ needed to reach this temperature, but this delay allows magnetic fields, caused by eddy currents induced when the coils switch off, to decay to $\lesssim 1 \mathrm{mG}$, which is needed for the subsequent microwave transfer step(s). The cloud expands slowly enough in the molasses that the density hardly changes during this period.

At this point, the population is distributed amongst the 12 Zeeman sublevels of ( $v=0, N=1)$ shown in Fig. 1(a) and the corresponding 12 levels in $v=1$. To purify the state distribution, we optically pump into $|1,0,0\rangle$ by reducing $I$ to $0.01 I_{\max }$ and increasing the rf power to the EOM so that the carrier, which addresses $F=0$, has $\leq 1 \%$ of the total power. This transfers $\epsilon_{\mathrm{OP}}=60 \%$ of the molecules to $F=0$, with the efficiency limited by imperfect extinction of the carrier and off-resonant excitation by other sidebands. It takes $40 \mu \mathrm{s}$ for the population to reach its new steady state, though we pump for $100 \mu \mathrm{s}$.

With the majority of molecules now in a single state, we transfer them to a selected Zeeman sublevel by driving 
microwave transitions between $N=0$ and $N=1$. The outputs of a two-channel microwave synthesizer are connected to a frequency doubler via a switch, and the doubled output is coupled to free space through a horn. The microwave field passes into the vacuum chamber along the same path as the slowing laser. Its polarization at the molecules is poorly defined due to reflections inside the chamber, and we find that $\Delta M_{F}=-1,0,1$ transitions can all be driven. Bias coils cancel the background magnetic field and apply a constant, uniform field of $\sim 60 \mathrm{mG}$, sufficient to resolve the Zeeman sublevels, but small enough not to disrupt the molasses cooling.

Figure 3(a) shows the depletion of the $N=1$ population as a function of microwave angular frequency, $\omega$, as it is scanned through the magnetically-insensitive $|1,0,0\rangle \rightarrow$ $|0,1,0\rangle$ transition at $\omega_{0}$. The microwave pulse duration is $\tau_{\mu 1}=140 \mu \mathrm{s}$, and the Rabi frequency is $\Omega=\pi / \tau_{\mu 1}$. Molecules transferred to $N=0$ are decoupled from the MOT light. We measure the number remaining in $N=1$ by turning the MOT back on and imaging the fluorescence after a time $\tau_{\text {hold }}$, typically $30 \mathrm{~ms}$. This number, divided by the initial number in the MOT, is the fraction recaptured. The line in Fig. 3(a) is a fit to the data using the model $y_{0}+A f\left(\Omega, \omega-\omega_{0}, \tau_{\mu 1}\right)$, where $y_{0}$ is the fraction recaptured without the microwave pulse, $A$ is an amplitude, and $f$ is the usual Rabi line shape for a two-level system. We fix $\tau_{\mu 1}$ and $\Omega$, leaving $y_{0}, A$, and $\omega_{0}$ as free parameters. The fit gives $y_{0}=0.57$, consistent with the MOT lifetime, and $A=-0.32$. The microwave transfer efficiency is $\epsilon_{\mathrm{MW}}=\left|A /\left(y_{0} \epsilon_{\mathrm{OP}}\right)\right|=94 \%$. We infer that, in the relevant polarization, the microwave intensity at the molecules is $64 \mathrm{nW} \mathrm{cm}^{-2}$. Figure 3(b) shows similar data for the magnetically-sensitive transition $|1,0,0\rangle \rightarrow|0,1,1\rangle$. We drive a $\pi$ pulse with a shorter duration of $\tau_{\mu 1}=40 \mu$ s to reduce the effects of magnetic field inhomogeneities and fluctuations. Their effects are still visible in the data, producing a slight broadening relative to the model, a poorer fit in the wings, and a lower efficiency of $\epsilon_{\mathrm{MW}}=87 \%$. The inferred intensity in the relevant polarization is $780 \mathrm{nW} \mathrm{cm}^{-3}$.

Figure 3(c) shows Rabi oscillations on the $|1,0,0\rangle \rightarrow$ $|0,1,1\rangle$ transition. We measure the percentage recaptured versus $\tau_{\mu 1}$, with $\omega$ on resonance and the power held constant. To model these data we found it necessary to include two imperfections. The first relates to the microwave synthesizer, which has a transient frequency drift when switched. This drift is well modeled by $\omega\left(t^{\prime}\right)=$ $\omega_{\infty}-\Delta \omega e^{-t^{\prime} / \tau}$, where $\omega_{\infty} /(2 \pi)$ is the frequency at long times, $t^{\prime}$ is the time since the start of the pulse, $\Delta \omega /(2 \pi) \approx$ $7 \mathrm{kHz}$ is the total frequency change, and $\tau \approx 105 \mu \mathrm{s}$ is the timescale. This has no observable effect on the line shapes in Figs. 3(a), 3(b), but it causes a slight frequency shift in the line center, a noticeable chirp in the frequency of the Rabi oscillations, and a slight reduction in their contrast. The second imperfection is due to gradients of intensity and polarization produced by the standing wave component of the microwave field, and it is the main reason for the gradual reduction in the contrast of the Rabi oscillations with increasing $\tau_{\mu 1}$. To model these effects, we solve the two-level optical Bloch equations with the measured frequency drift included, producing a function $y_{0}+$ $A g\left(\Omega, \omega_{\infty}-\omega_{0}, \tau_{\mu 1}\right)$. We average this over a Gaussian distribution of Rabi frequencies with a width of $\Delta \Omega$. The solid line in Fig. 3(c) is a fit to this model, with $y_{0}, A$, $\omega_{\infty}-\omega_{0}, \Omega$, and $\Delta \Omega$ as free parameters. We find $\Delta \Omega / \Omega=0.16$, which is reasonable since the distance from (a)

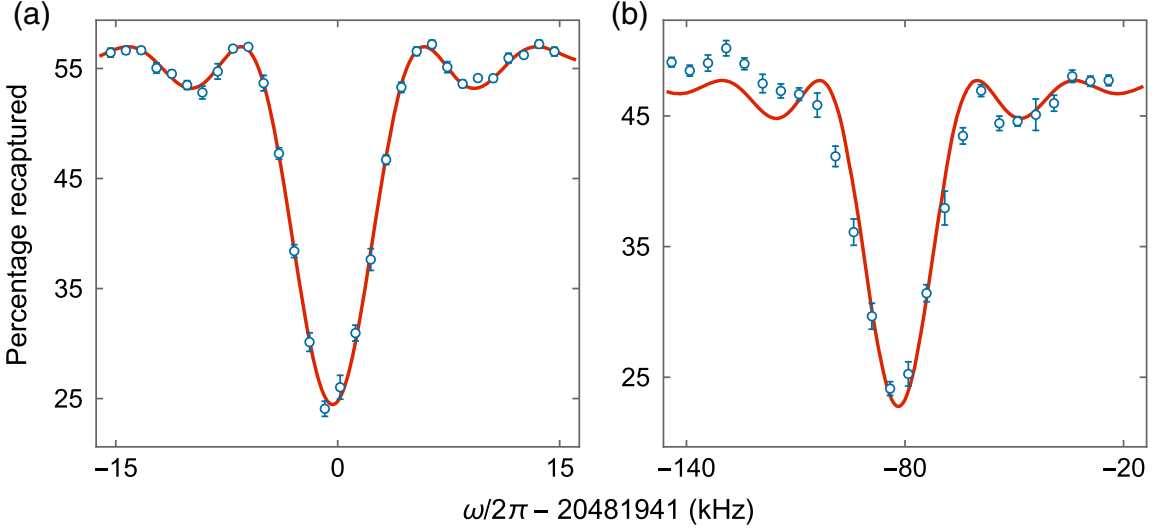

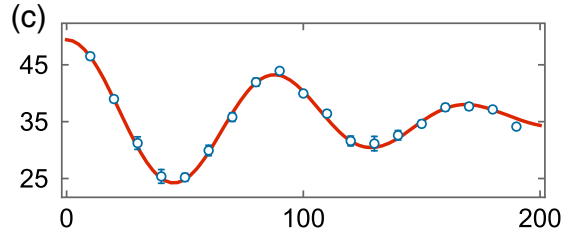

(d)

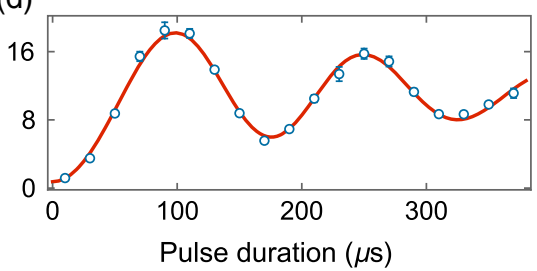

FIG. 3. Microwave transitions between rotational levels. (a),(b) The depletion of $N=1$ population versus microwave frequency: (a) $|1,0,0\rangle \rightarrow|0,1,0\rangle$ transition driven by a $140 \mu \mathrm{s} \pi$ pulse; (b) $|1,0,0\rangle \rightarrow|0,1,1\rangle$ transition driven by a $40 \mu$ s $\pi$ pulse. (c) Rabi oscillations driven between $|1,0,0\rangle$ and $|0,1,1\rangle$. (d) Rabi oscillations between $|0,1,1\rangle$ and $|1,2,2\rangle$ after a push pulse has removed all molecules in $N=1$. Each point gives the average and standard error of nine experiments. Where not visible, error bars are smaller than points. In (a),(b), lines show fits to the Rabi line shape for a $\pi$ pulse of the known duration, with background, amplitude and central frequency as free parameters. The data have been corrected for a systematic frequency shift of $1.9 \mathrm{kHz}$ in (a) and $4.7 \mathrm{kHz}$ in (b), arising from a drift in microwave frequency when the pulse is applied. In (c),(d), lines show fits to solutions of the optical Bloch equations with time varying detuning and integration over Rabi frequencies (see text). 
node to antinode of the standing wave is $3.5 \mathrm{~mm}$, comparable to the size of the molecule cloud.

With most molecules now transferred to $N=0$, we push those remaining in $N=1$ out of the trap region by turning on the slowing light for $1 \mathrm{~ms}$. This leaves a pure sample of molecules in a single state. We then either turn on the magnetic trap or apply a second microwave pulse, of duration $\tau_{\mu 2}$, to transfer back to a selected sublevel of $N=1$. Figure 3(d) shows Rabi oscillations on the $|0,1,1\rangle \rightarrow|1,2,2\rangle$ transition as $\tau_{\mu 2}$ is varied. The percentage recaptured is zero when $\tau_{\mu 2}=0$, showing that we indeed have a pure sample. The line is a fit using the same model described above. A $\pi$ pulse takes $100 \mu$ s, implying a microwave intensity of $42 \mathrm{nW} \mathrm{cm}{ }^{-2}$, and it has an efficiency of $75 \%$.

With these procedures, we can trap molecules in any of the weak-field seeking states shown in Fig. 1(a). Here, we demonstrate trapping in the $|0,1,1\rangle$ and $|1,2,2\rangle$ states. We turn the trap on with $B^{\prime}=30 \mathrm{G} \mathrm{cm}^{-1}$ after the first or second microwave pulse, then detect the number remaining after a time $\tau_{\text {mag }}$ by turning the MOT light on and imaging the fluorescence. When trapping molecules in $N=0$, which do not fluoresce in the MOT light, we transfer back to $N=1$ using the $|0,1,1\rangle \rightarrow|1,2,2\rangle$ transition prior to detection. This magnetically-insensitive transition can be driven while the molecules are magnetically trapped. Indeed, we observe Rabi oscillations on this transition, similar to those in Fig. 3(d), even when the molecules are trapped. The number of trapped molecules follows a singleexponential decay, $N_{\text {mol }}\left(\tau_{\text {mag }}\right)=N_{\text {mol }}(0) \exp \left(-R_{\text {loss }} \tau_{\text {mag }}\right)$, which we attribute mainly to collisions with helium from the buffer-gas source. Figure 4 shows the loss rate, $R_{\text {loss }}$, versus helium flow rate, for molecules in each of the two states, showing a linear dependence in both cases. The

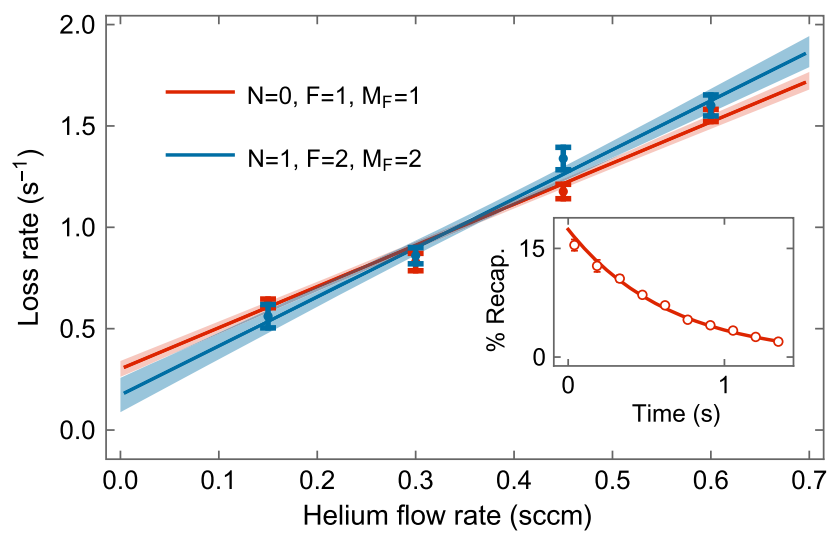

FIG. 4. The magnetic trap loss rate versus the helium flow rate for molecules in states $|0,1,1\rangle$ (red) and $|1,2,2\rangle$ (blue). Lines: linear fits. Shaded regions: $68 \%$ confidence bands. Inset: The percentage recaptured versus the time in the magnetic trap for molecules in $|0,1,1\rangle$, when flow rate is $0.6 \mathrm{sccm}$. Where not visible, error bars are smaller than points. gradients are 2.03(8) and 2.42(16) s $\mathrm{s}^{-1} \mathrm{sccm}^{-1}$ for the $|0,1,1\rangle$ and $|1,2,2\rangle$ states, respectively, differing by 2.2 $\sigma$. Extrapolating to zero flow, the loss rates are 0.30 (3) and $0.17(6) \mathrm{s}^{-1}$, differing by $1.9 \sigma$. These rates are close to the loss rate due to vibrational excitation by room temperature blackbody radiation [38], which is $0.22 \mathrm{~s}^{-1}$ for both states. At the lowest flow used, $0.15 \mathrm{sccm}$, the trap lifetimes are 1.60(6) s for $|0,1,1\rangle$ and 1.8(2) s for $|1,2,2\rangle$.

Before turning on the trap, the radial, axial, and geometric mean temperatures of the molecules are $\left\{T_{\rho}, T_{z}, T\right\}=$ $\{52(12), 69(12), 57(9)\} \mu \mathrm{K}$. After trapping for $\tau_{\text {mag }}=40 \mathrm{~ms}$, we measure $\{56(12), 131(12), 74(11)\} \mu \mathrm{K}$, while at $\tau_{\text {mag }}=$ $500 \mathrm{~ms}$, the values are $\{47(12), 123(12), 65(11)\} \mu \mathrm{K}$. These measurements show that trap loading results in modest heating, though only in the axial direction, and that the heating rate in the trap is consistent with zero and has an upper limit of $34 \mu \mathrm{K} \mathrm{s}^{-1}$. Taking the mean of the two measurements gives a temperature for the trapped molecules of $70(8) \mu \mathrm{K}$. We load about $5 \times 10^{3}$ molecules into the trap, and the cloud has radial and axial rms radii of $\sigma_{\rho}=1.37(1) \mathrm{mm}$ and $\sigma_{z}=1.44(2) \mathrm{mm}$. The density is $1.2 \times 10^{5} \mathrm{~cm}^{-3}$, and it could be increased by increasing $B^{\prime}$. The phase-space density is $2.6 \times 10^{-12}$.

In summary, we have compressed our MOT to increase the density of molecules, demonstrated coherent control of their rotational, hyperfine and magnetic states, and transferred them to a conservative trap. We have measured the trap loss rate for two selected states, which is a prototype for future studies of elastic and inelastic collisions between cotrapped ultracold atoms and state-selected molecules [39]. Our demonstrations of long lifetimes and low heating rates in a magnetic trap are important for reaching lower temperatures by sympathetic cooling [40,41]. The magnetically trapped molecules can now easily be transported to experiments more conveniently located away from the MOT region [42]. The quantum state control we demonstrate is required for measurements with ultracold molecules that test fundamental physics [43-46]. With sufficient control, quantum information can be stored within hyperfine states and the dipole-dipole interaction turned on when needed for information processing [6]. Controlled microwave-induced dipoles are important for simulating spin Hamiltonians $[7,10,47,48]$, studying topological superfluids [49], and enhancing evaporative cooling [50]. For induced dipoles of $\mu_{e} / \sqrt{3}$, where $\mu_{e}=3.07 \mathrm{D}$ is the dipole moment of $\mathrm{CaF}$ [51], and taking a realistic spacing of $0.5 \mu \mathrm{m}$, the dipole-dipole interaction energy is a few $\mathrm{kHz}$, comparable to the resolution we achieve. Thus, our work demonstrates many of the key capabilities needed for the applications of laser-cooled molecules.

Underlying data may be accessed from Zenodo [52] and used under the Creative Commons CCZero license.

We are grateful to J. Dyne, G. Marinaro and V. Gerulis for technical assistance. The research has received funding 
from EPSRC under Grants No. EP/M027716/1 and No. EP/ P01058X/1, and from the European Research Council under the European Union's Seventh Framework Programme (FP7/2007-2013)/ERC Grant agreement No. 320789.

H. J. Williams and L. Caldwell contributed equally to this work.

*m.tarbutt@imperial.ac.uk

[1] M. S. Safronova, D. Budker, D. DeMille, D. F. Jackson Kimball, A. Derevianko, and C.W. Clark, Search for new physics with atoms and molecules, arXiv: 1710.01833.

[2] D. DeMille, J. M. Doyle, and A. O. Sushkov, Probing the frontiers of particle physics with tabletop-scale experiments, Science 357, 990 (2017).

[3] R. V. Krems, Cold controlled chemistry, Phys. Chem. Chem. Phys. 10, 4079 (2008).

[4] D. DeMille, Quantum Computation with Trapped Polar Molecules, Phys. Rev. Lett. 88, 067901 (2002).

[5] S. F. Yelin, K. Kirby, and R. Coté, Schemes for robust quantum computation with polar molecules, Phys. Rev. A 74, 050301(R) (2006).

[6] A. André, D. DeMille, J. M. Doyle, M. D. Lukin, S. E. Maxwell, P. Rabl, R. J. Schoelkopf, and P. Zoller, A coherent all-electrical interface between polar molecules and mesoscopic superconducting resonators, Nat. Phys. 2, 636 (2006).

[7] A. Micheli, G. K. Brennen, and P. Zoller, A toolbox for lattice-spin models with polar molecules, Nat. Phys. 2, 341 (2006).

[8] D. Barredo, S. de Léséleuc, V. Lienhard, T. Lahaye, and A. Browaeys, An atom-by-atom assembler of defect- free arbitrary 2d atomic arrays, Science 354, 1021 (2016).

[9] T. Lahaye, C. Menotti, L. Santos, M. Lewenstein, and T. Pfau, The physics of dipolar bosonic quantum gases, Rep. Prog. Phys. 72, 126401 (2009).

[10] B. Yan, S. A. Moses, B. Gadway, J. P. Covey, K. R. A. Hazzard, A. M. Rey, D. S. Jin, and J. Ye, Observation of dipolar spin-exchange interactions with lattice-confined polar molecules, Nature (London) 501, 521 (2013).

[11] S. A. Will, J. W. Park, Z.Z. Yan, H. Loh, and M. W. Zwierlein, Coherent Microwave Control of Ultracold ${ }^{23} \mathrm{Na}^{40} \mathrm{~K}$ Molecules, Phys. Rev. Lett. 116, 225306 (2016).

[12] P. D. Gregory, J. Aldegunde, J. M. Hutson, and S. L. Cornish, Controlling the rotational and hyperfine state of ultracold ${ }^{87} \mathrm{Rb}^{133} \mathrm{Cs}$ molecules, Phys. Rev. A 94, 041403(R) (2016).

[13] J. W. Park, Z.Z. Yan, H. Loh, S. A. Will, and M. W. Zwierlein, Second-scale nuclear spin coherence time of ultracold ${ }^{23} \mathrm{Na}^{40} \mathrm{~K}$ molecules, Science 357, 372 (2017).

[14] K.-K. Ni, S. Ospelkaus, M. H. G. de Miranda, A. Peér, B. Neyenhuis, J. J. Zirbel, S. Kotochigova, P. S. Julienne, D. S. Jin, and J. Ye, A high phase-space-density gas of polar molecules, Science 322, 231 (2008).

[15] T. Takekoshi, L. Reichsöllner, A. Schindewolf, J. M. Hutson, C. R. Le Sueur, O. Dulieu, F. Ferlaino, R. Grimm, and H.-C. Nägerl, Ultracold Dense Samples of Dipolar
RbCs Molecules in the Rovibrational and Hyperfine Ground State, Phys. Rev. Lett. 113, 205301 (2014).

[16] P. K. Molony, P. D. Gregory, Z. Ji, B. Lu, M. P. Köppinger, C. R. Le Sueur, C. L. Blackley, J. M. Hutson, and S. L. Cornish, Creation of Ultracold ${ }^{87} \mathrm{Rb}^{133} \mathrm{Cs}$ Molecules in the Rovibrational Ground State, Phys. Rev. Lett. 113, 255301 (2014).

[17] J. W. Park, S. A. Will, and M. W. Zwierlein, Ultracold Gas of Fermionic ${ }^{23} \mathrm{Na}^{40} \mathrm{~K}$ Molecules in their Absolute Ground State, Phys. Rev. Lett. 114, 205302 (2015).

[18] M. Guo, B. Zhu, B. Lu, X. Ye, F. Wang, R. Vexiau, N. Bouloufa-Maafa, G. Quéméner, O. Dulieu, and D. Wang, Creation of an Ultracold Gas of Ground-State Dipolar ${ }^{23} \mathrm{Na}^{87} \mathrm{Rb}$ Molecules, Phys. Rev. Lett. 116, 205303 (2016).

[19] A. Prehn, M. Ibrügger, R. Glöckner, G. Rempe, and M. Zeppenfeld, Optoelectrical Cooling of Polar Molecules to Submillikelvin Temperatures, Phys. Rev. Lett. 116, 063005 (2016).

[20] J. F. Barry, D. J. McCarron, E. B. Norrgard, M. H. Steinecker, and D. DeMille, Magneto-optical trapping of a diatomic molecule, Nature (London) 512, 286 (2014).

[21] D. J. McCarron, E. B. Norrgard, M. H. Steinecker, and D. DeMille, Improved magneto-optical trapping of a diatomic molecule, New J. Phys. 17, 035014 (2015).

[22] M. H. Steinecker, D. J. McCarron, Y. Zhu, and D. DeMille, Improved radio-frequency magneto-optical trap of $\mathrm{SrF}$ molecules, ChemPhysChem 17, 3664 (2016).

[23] S. Truppe, H. J. Williams, M. Hambach, L. Caldwell, N. J. Fitch, E. A. Hinds, B. E. Sauer, and M. R. Tarbutt, Molecules cooled below the Doppler limit, Nat. Phys. 13, 1173 (2017).

[24] L. Anderegg, B. L. Augenbraun, E. Chae, B. Hemmerling, N. R. Hutzler, A. Ravi, A. Collopy, J. Ye, W. Ketterle, and J. M. Doyle, Radio Frequency Magneto-Optical Trapping of CaF with High Density, Phys. Rev. Lett. 119, 103201 (2017).

[25] H. J. Williams, S. Truppe, M. Hambach, L. Caldwell, N. J. Fitch, E. A. Hinds, B. E. Sauer, and M. R. Tarbutt, Characteristics of a magneto-optical trap of molecules, New J. Phys. 19, 113035 (2017).

[26] J. D. Weinstein, R. deCarvalho, T. Guillet, B. Friedrich, and J. M. Doyle, Magnetic trapping of calcium monohydride molecules at millikelvin temperatures, Nature (London) 395, 148 (1998).

[27] B. C. Sawyer, B. L. Lev, E. R. Hudson, B. K. Stuhl, M. Lara, J. L. Bohn, and J. Ye, Magnetoelectrostatic Trapping of Ground State OH Molecules, Phys. Rev. Lett. 98, 253002 (2007).

[28] S. D. Hogan, A. W. Wiederkehr, H. Schmutz, and F. Merkt, Magnetic Trapping of Hydrogen After Multistage Zeeman Deceleration, Phys. Rev. Lett. 101, 143001 (2008).

[29] E. Tsikata, W. C. Campbell, M. T. Hummon, H. I. Lu, and J. M. Doyle, Magnetic trapping of NH molecules with $20 \mathrm{~s}$ lifetime, New J. Phys. 12, 065028 (2010).

[30] J. Riedel, S. Hoekstra, W. Jäger, J. J. Gilijamse, S. Y. T. van de Meerakker, and G. Meijer, Accumulation of Starkdecelerated NH molecules in a magnetic trap, Eur. Phys. J. D 65, 161 (2011).

[31] Hsin-I Lu, I. Kozyryev, B. Hemmerling, J. Piskorski, and J. M. Doyle, Magnetic Trapping of Molecules via Optical Loading and Magnetic Slowing, Phys. Rev. Lett. 112, 113006 (2014). 
[32] N. Akerman, M. Karpov, Y. Segev, N. Bibelnik, J. Narevicius, and E. Narevicius, Trapping of Molecular Oxygen Together with Lithium Atoms, Phys. Rev. Lett. 119, 073204 (2017).

[33] Y. Liu, M. Vashishta, P. Djuricanin, S. Zhou, W. Zhong, T. Mittertreiner, D. Carty, and T. Momose, Magnetic Trapping of Cold Methyl Radicals, Phys. Rev. Lett. 118, 093201 (2017).

[34] S. Truppe, M. Hambach, S. M. Skoff, N. E. Bulleid, J. S. Bumby, R. J. Hendricks, E. A. Hinds, B. E. Sauer, and M. R. Tarbutt, A buffer gas beam source for short, intense and slow molecular pulses, J. Mod. Opt. 65, 648 (2018).

[35] S. Truppe, H. J. Williams, N. J. Fitch, M. Hambach, T. E. Wall, E. A. Hinds, B. E. Sauer, and M. R. Tarbutt, An intense, cold, velocity-controlled molecular beam by frequencychirped laser slowing, New J. Phys. 19, 022001 (2017).

[36] M. R. Tarbutt and T. C. Steimle, Modeling magnetooptical trapping of $\mathrm{CaF}$ molecules, Phys. Rev. A 92, 053401 (2015).

[37] W. Petrich, M. H. Anderson, J. R. Ensher, and E. A. Cornell, Behavior of atoms in a compressed magneto-optical trap, J. Opt. Soc. Am. B 11, 1332 (1994).

[38] S. Y. Buhmann, M. R. Tarbutt, S. Scheel, and E. A. Hinds, Surface-induced heating of cold polar molecules, Phys. Rev. A 78, 052901 (2008).

[39] L. P. Parazzoli, N. J. Fitch, P. S. Żuchowski, J. M. Hutson, and H. J. Lewandowski, Large Effects of Electric Fields on Atom-Molecule Collisions at Millikelvin Temperatures, Phys. Rev. Lett. 106, 193201 (2011).

[40] S. K. Tokunaga, W. Skomorowski, P. S. Żuchowski, R. Moszynski, J. M. Hutson, E. A. Hinds, and M. R. Tarbutt, Prospects for sympathetic cooling of molecules in electrostatic, ac and microwave traps, Eur. Phys. J. D 65, 141 (2011).

[41] J. Lim, M. D. Frye, J. M. Hutson, and M. R. Tarbutt, Modeling sympathetic cooling of molecules by ultracold atoms, Phys. Rev. A 92, 053419 (2015).
[42] H. J. Lewandowski, D. M. Harber, D. L. Whitaker, and E. A. Cornell, Simplified system for creating a Bose-Einstein condensate, J. Low Temp. Phys. 132, 309 (2003).

[43] M. R. Tarbutt, B. E. Sauer, J. J. Hudson, and E. A. Hinds, Design for a fountain of $\mathrm{YbF}$ molecules to measure the electron's electric dipole moment, New J. Phys. 15, 053034 (2013).

[44] L. R. Hunter, S. K. Peck, A. S. Greenspon, S. S. Alam, and D. DeMille, Prospects for laser cooling TIF, Phys. Rev. A 85, 012511 (2012).

[45] S. B. Cahn, J. Ammon, E. Kirilov, Y. V. Gurevich, D. Murphree, R. Paolino, D. A. Rahmlow, M. G. Kozlov, and D. DeMille, Zeeman-Tuned Rotational-Level Crossing Spectroscopy in a Diatomic Free Radical, Phys. Rev. Lett. 112, 163002 (2014).

[46] C. Cheng, A. P. P. van der Poel, P. Jansen, M. Quintero-Pérez, T. E. Wall, W. Ubachs, and H. L. Bethlem, Molecular Fountain, Phys. Rev. Lett. 117, 253201 (2016).

[47] R. Barnett, D. Petrov, M. Lukin, and E. Demler, Quantum Magnetism with Multicomponent Dipolar Molecules in an Optical Lattice, Phys. Rev. Lett. 96, 190401 (2006).

[48] A. V. Gorshkov, S. R. Manmana, G. Chen, J. Ye, E. Demler, M. D. Lukin, and A. M. Rey, Tunable Superfluidity and Quantum Magnetism with Ultracold Polar Molecules, Phys. Rev. Lett. 107, 115301 (2011).

[49] N. R. Cooper and G. V. Shlyapnikov, Stable Topological Superfluid Phase of Ultracold Polar Fermionic Molecules, Phys. Rev. Lett. 103, 155302 (2009).

[50] A. V. Avdeenkov, Dipolar collisions of ultracold polar molecules in a microwave field, Phys. Rev. A 86, 022707 (2012).

[51] W. J. Childs, L. S. Goodman, U. Nielsen, and V. Pfeufer, Electric dipole moment of $\mathrm{CaF}\left(\mathrm{X}^{2} \Sigma^{+}\right)$by molecular beam, laser-rf, double-resonance study of Stark splittings, J. Chem. Phys. 80, 2283 (1984).

[52] https://doi.org/10.5281/zenodo.1035877. 